\title{
Spinal cord MRI and MRS Detect Early-stage Alterations and Disease Progression in Friedreich Ataxia
}

James M. Joers ${ }^{1 *}$, Isaac M. Adanyeguh ${ }^{1 *}$, Dinesh K. Deelchand ${ }^{1}$, Diane H. Hutter ${ }^{1}$, Lynn E. Eberly $^{2}$, Isabelle Iltis ${ }^{1}$, Khalaf O. Bushara $^{3}$, Christophe Lenglet ${ }^{1 \dagger}$, Pierre-Gilles Henry ${ }^{1 \dagger}$

${ }^{1}$ Center for Magnetic Resonance Research and Department of Radiology, University of Minnesota Medical School, Minneapolis, MN, USA

${ }^{2}$ Division of Biostatistics, School of Public Health, University of Minnesota, Minneapolis, MN, USA

${ }^{3}$ Department of Neurology, University of Minnesota Medical School, Minneapolis, MN, USA

* J.M.J. and I.M.A. contributed equally and share the first authorship

$\dagger$ C.L. and P-G.H. contributed equally and share the last authorship

\section{Correspondance to:}

Pierre-Gilles Henry, Ph.D.

Associate Professor

Center for Magnetic Resonance Research

$20216^{\text {th }}$ Street SE

Minneapolis MN 55455

henry@,cmrr.umn.edu

Running title: Spinal cord MRI and MRS in Friedreich Ataxia (37 chars, max 40)

Keywords: magnetic resonance, spectroscopy, diffusion imaging, spinal cord, Friedreich Ataxia, longitudinal

\section{Abbreviations}

9HPT $=9$-hole peg test 
$\mathrm{AD}=$ axial diffusivity

$\mathrm{ADL}=$ activities of daily living

$\mathrm{BL}=$ baseline

$\mathrm{Cr}=$ creatine

CRLB $=$ Cramer-Rao lower bounds

$\mathrm{CSA}=$ cross-sectional area

$\mathrm{CSF}=$ cerebrospinal fluid

$\mathrm{dMRI}=$ diffusion MRI

$\mathrm{EPI}=$ echo-planar imaging

$\mathrm{FA}=$ fractional anisotropy

$\mathrm{FARS}=$ Friedreich ataxia rating scale

FRDA $=$ Friedreich Ataxia

$\mathrm{GPC}=$ glycerophosphocholine

$\mathrm{MD}=$ mean diffusivity

$\mathrm{mIns}=$ myo-inositol

$\mathrm{MRS}=$ magnetic resonance spectroscopy

$\mathrm{NAA}=\mathrm{N}$-acetyl-aspartate

NAAG $=\mathrm{N}$-acetyl-aspartyl-glutamate

PCho $=$ phosphocholine

$\mathrm{PCr}=$ phosphocreatine

$\mathrm{RD}=$ radial diffusivity

$\mathrm{SARA}=$ scale for the assessment and rating of ataxia

$\mathrm{SCT}=$ spinal cord toolbox

$\mathrm{SNR}=$ signal-to-noise ratio

$\mathrm{SRM}=$ standardized response mean

tCho $=$ total choline

$\mathrm{tCr}=$ total creatine

tNAA $=$ total $\mathrm{N}$-acetyl-aspartate 


\begin{abstract}
Background: Friedreich Ataxia (FRDA) is the most common hereditary ataxia. Atrophy of the spinal cord is one of the hallmarks of the disease. Magnetic resonance imaging (MRI) and spectroscopy (MRS) are powerful and non-invasive tools to investigate pathological changes in the spinal cord. A handful of studies have reported cross-sectional alterations in FRDA using MRI and diffusion MRI (dMRI) in FRDA. However, to our knowledge no longitudinal MRI, dMRI or MRS results have been reported in the spinal cord in FRDA.
\end{abstract}

Objective: To investigate early-stage cross-sectional alterations and longitudinal changes in the cervical spinal cord in FRDA, using a multimodal magnetic resonance (MR) protocol comprising morphometric (anatomical MRI), microstructural (dMRI), and neurochemical ( ${ }^{1} \mathrm{H}$ MRS) assessments.

Design: We enrolled 28 early-stage individuals with FRDA and 20 age- and gender-matched controls (cross-sectional study). Disease duration at baseline was $5.5 \pm 4.0$ years and Friedreich Ataxia Rating Scale (FARS) total neurological score at baseline was $42.7 \pm 13.6$. Twenty-one FRDA participants returned for 1-year follow-up, and 19 of those for 2-year follow-up (cohort study). Each visit consisted in clinical assessments and MR scans. Controls were scanned at baseline only.

Results: At baseline, individuals with FRDA had significantly lower spinal cord cross-sectional area $\left(-31 \%, p=4.10^{-17}\right)$, higher eccentricity $\left(+10 \%, p=5.10^{-7}\right)$, lower total $\mathrm{N}$-acetyl-aspartate $(-36 \%$, $\left.\mathrm{p}=6.10^{-9}\right)$ and higher myo-inositol $\left(+37 \%, \mathrm{p}=2.10^{-6}\right)$ corresponding to a lower ratio tNAA/mIns ($\left.52 \%, \mathrm{p}=2.10^{-13}\right)$, lower fractional anisotropy $\left(-24 \%, \mathrm{p}=10^{-9}\right)$ as well as higher radial diffusivity $\left(+56 \%, \mathrm{p}=2.10^{-9}\right)$, mean diffusivity $\left(+35 \%, \mathrm{p}=10^{-8}\right)$ and axial diffusivity $\left(+17 \%, \mathrm{p}=4.10^{-5}\right)$ relative to controls.

Longitudinally, spinal cord cross-sectional area decreased by $2.4 \%$ per year relative to baseline $\left(\mathrm{p}=4.10^{-4}\right)$, the ratio $\mathrm{tNAA} / \mathrm{mIns}$ decreased by $5.8 \%$ per year $(\mathrm{p}=0.03)$, and fractional anisotropy showed a trend to decrease $(-3.2 \%$ per year, $\mathrm{p}=0.08)$. 
Spinal cord cross-sectional area correlated strongly with clinical measures, with the strongest correlation coefficients found between cross-sectional area and Scale for the Assessment and Rating of Ataxia (SARA) $\left(\mathrm{R}=-0.55, \mathrm{p}=7.10^{-6}\right)$ and between cross-sectional area and FARS total neurological score $\left(R=-0.60, p=4 \cdot 10^{-7}\right)$. Less strong but still significant correlations were found for fractional anisotropy and $\mathrm{tNAA} / \mathrm{mIns}$.

Conclusion: We report here the first quantitative longitudinal MR results in the spinal cord in FRDA. The largest longitudinal effect size was found for spinal cord cross-sectional area, followed by tNAA/mIns and fractional anisotropy. Our results provide direct evidence that abnormalities in the spinal cord result not solely from hypoplasia, but also from neurodegeneration, and show that disease progression can be monitored non-invasively in the spinal cord. 


\section{INTRODUCTION}

Friedreich ataxia (FRDA) is the most common inherited ataxia, with an incidence ranging from 1:20,000 to 1:250,000, or less depending on countries ${ }^{1,2}$. Genetic transmission is autosomal recessive and is caused by mutations in the frataxin gene (in most cases a GAA repeat expansion in intron 1) leading to reduced expression of frataxin ${ }^{3,4}$. Symptoms include limb and gait ataxia, dysarthria and cardiomyopathy ${ }^{5}$. Onset is typically in the second decade of life ${ }^{6}$ and there is currently no effective disease-modifying treatment.

Nikolaus Friedreich recognized spinal cord degeneration as a hallmark of the disease ${ }^{7}$ and spinal cord atrophy was reported in early MRI studies ${ }^{8-12}$. In the pathogenesis of FRDA, substantial demyelination and gliosis of the posterior and lateral columns of the spinal cord occur prior to cerebellar pathology ${ }^{5}$. In particular, dorsal root ganglia, posterior roots and posterior columns of the spinal cord show significant pathological alterations ${ }^{13-15}$. Recent cross-sectional MRI studies showed that spinal cord cross-sectional area (CSA) is significantly smaller in FRDA than in controls and correlates negatively with disease severity ${ }^{15-17}$. However, longitudinal MR data documenting disease progression in the spinal cord are still lacking. With many clinical trials ongoing or on the horizon, including gene therapy trials, MR could play a key role in assessing disease progression and the effect of prospective treatments. Consequently, "natural history" longitudinal MR data documenting disease progression in the spinal cord are urgently needed.

Beyond anatomical imaging, advanced MR modalities, such as diffusion MRI (dMRI) and magnetic resonance spectroscopy (MRS) have the potential to respectively reveal microstructural and neurochemical alterations not visible in conventional anatomical images.

Diffusion MRI ${ }^{18}$ relies on the anisotropic diffusion of water in organized tissues, such as the brain white matter or spinal cord, to recover microstructural and connectivity information through local biophysical modeling and tractography ${ }^{19}$. Axonal membranes hinder the diffusion of water molecules, a phenomenon that can be quantified through MRI by taking measurements along multiple orientations. Diffusion tensor imaging (DTI) models diffusion MRI data by assuming Gaussian diffusion at each location of the imaged tissue. Diffusion MRI of the spinal cord is 
challenging and has only recently received growing attention and seen new developments in the neuroimaging community ${ }^{20-26}$, with applications in disorders such as degenerative cord compression ${ }^{27}$, multiple sclerosis ${ }^{28}$, amyotrophic lateral sclerosis ${ }^{23}$ and traumatic spinal cord injury ${ }^{29}$. To the best of our knowledge, only one study has reported cross-sectional data using dMRI in the spinal cord in FRDA ${ }^{30}$, and no longitudinal dMRI data have been published.

Proton Magnetic Resonance Spectroscopy ( $\left.{ }^{1} \mathrm{H}-\mathrm{MRS}\right)$ allows non-invasive measurement of the concentration of multiple metabolites in multiple organs, including the spinal cord. Although

challenging to implement, ${ }^{1} \mathrm{H}-\mathrm{MRS}$ in the spinal cord has been shown to be feasible ${ }^{31-34}$. A number of studies have reported neurochemical alterations in the spinal cord in neurodegenerative diseases (Blamire, Cader et al. 2007, Marliani, Clementi et al. 2010, Elliott, Pedler et al. 2011), and changes in N-acetyl-aspartate (NAA) concentrations in a longitudinal assessment of lesions of the spinal cord in multiple sclerosis (Ciccarelli, Altmann et al. 2010). However, no data have been reported using MRS in the spinal cord in FRDA.

The objective of the present study was to study pathological alterations and longitudinal changes in the cervical spinal cord in an early-stage cohort of individuals with FRDA, using an advanced multimodal MR protocol (MRI, dMRI and MRS). We hypothesized that FRDA participants would show significant alterations in morphometry, microstructure and neurochemistry relative to controls, even at early-stage, and that FRDA participants would show changes over time.

\section{MATERIALS AND METHODS}

\section{Participants}

All procedures were conducted in accordance with the Declaration of Helsinki and were approved by the University of Minnesota Institutional Review Board. All participants provided informed consent (adults) or, for minors, informed assent with their parents providing informed consent.

Twenty-eight individuals with FRDA (mean age $19.0 \pm 7.3$ years, range 11-35, 14M/14F) and 20 age- and gender-matched control individuals (20.4 \pm 7.1 years, range 10-35, 11M/9F) were 
recruited for this study (Table 1). The FRDA group was at an early stage of the disease (Time from diagnosis: $2.3 \pm 2.6$ years; Disease duration: $5.5 \pm 4.0$ years). FRDA participants were recruited through the University of Minnesota Ataxia Research Center and the Friedreich's Ataxia Research Alliance (FARA). Control participants were recruited through the local CMRR volunteer list and word of mouth. Eligibility criteria for FRDA participants were: confirmed FRDA diagnosis. Efforts were made to primarily enroll FRDA participants at an early-stage of the disease. Only two FRDA participants were wheelchair-bound at baseline. Exclusion criteria for controls were: neurological disease. Exclusion criteria for all participants were: contraindication for MR scan, pregnancy, claustrophobia, diabetes, smoking, restless leg syndrome, history of alcoholism. All subjects were asked to withhold antioxidants such as multivitamins for 3 weeks prior to scanning. All subjects were also asked to withhold benzodiazepine or any medication specific to ataxia symptoms for 36 hours prior to scanning.

Twenty-one participants with FRDA returned for follow-up (referred to as the "longitudinal cohort") approximately one year later (time between baseline and 1-year follow-up =13.7 \pm 2.2 months), and 19 participants also returned for a 2-year follow-up (time between baseline and 2year follow-up $=26.8 \pm 3.4$ months). In the following, we refer to "1-year" and "2-year" followups for brevity. Controls were scanned only at baseline. Reasons for not returning for 1-year and 2-year follow-up included: too progressed (1), rod placement (2), brain arteriovenous anomaly (1), claustrophobia (1) and no longer interested or too busy (4).

Study visits took place at the Center for Magnetic Resonance Research, University of Minnesota from 2013 to 2018. Each visit included three 1 hr scanning sessions at 3 Tesla over 1-2 days: One for brain anatomical and diffusion MRI, one for spinal cord dMRI, and one for spinal cord MRS. Because of the large amount of data, we focus in this paper on the spinal cord findings, with the brain anatomical MRI used only for cervical spinal cord morphometry.

In addition to MR scans, all patients were assessed with the original Friedreich Ataxia Rating Scale (FARS $)^{35}$, which includes total neurological score (FARS total neuro, range 0-117), activities of daily living (ADL, range 0-36), functional staging (range 0-6), timed 9-hole peg test (9HPT) with dominant and non-dominant hand and timed $25 \mathrm{ft}$ walk test. In addition, Scale for the Assessment 
and Rating of Ataxia (SARA) scores (range 0-40) were collected except for 15 FRDA participants at baseline.

\section{MR Data Acquisition}

Measurements were initially performed on a 3 Tesla Siemens MAGNETOM Trio scanner (Siemens, Erlangen, Germany) with Syngo software version VD13D. The Trio scanner was upgraded to a MAGNETOM Prisma ${ }^{\text {fit }}$ scanner with Syngo software version VE11C during the study. The potential impact of this upgrade was carefully investigated and additional scans and analyses were performed to ensure that the upgrade did not bias our results (see "Scanner upgrade" section below).

On both scanners, the body transmit RF coil was used. On Trio, a 32-channel receive head coil was used for brain acquisitions, and a 20-channel receive head-neck coil was used for spine acquisitions. On Prisma, a 64-channel receive head-neck coil was used for all acquisitions.

\section{Brain Anatomical MRI}

Brain coronal $\mathrm{T}_{1}$-weighted 3D MPRAGE images were obtained with the following parameters: voxel size $1 \mathrm{~mm}$ isotropic, $\mathrm{TR} / \mathrm{TE}=2530 / 3.65 \mathrm{~ms}$, $\mathrm{TI}=1100 \mathrm{~ms}$, flip angle $=7^{\circ}, 224$ slices, field of view $=256 \times 176 \mathrm{~mm}^{2}$, phase encode in R-L direction, 2x GRAPPA.

\section{Spinal Cord Diffusion MRI}

Sagittal $\mathrm{T}_{2}$-weighted 2D TSE images with low resolution in the slice direction were acquired for positioning the dMRI volume of interest and for identification of cervical levels, with the following parameters: field of view 260x260 mm², matrix 384x384, 13 slices, $3 \mathrm{~mm}$ slice thickness, $0.7 \times 0.7$ $\mathrm{mm}^{2}$ in-plane resolution, $\mathrm{TR} / \mathrm{TE}=3500 / 112 \mathrm{~ms}$, flip angle $160 \mathrm{deg}$.

The cervical spine was scanned axially, covering levels $\mathrm{C} 2$ to $\mathrm{C} 7$, with $1.12 \times 1.12 \mathrm{~mm}^{2}$ (field of view $118 \times 62 \mathrm{~mm}^{2}$ ) in-plane resolution and $3.3 \mathrm{~mm}$ slice thickness (30 slices), as illustrated in Figure 2. A segmented-readout EPI sequence (RESOLVE) ${ }^{36}$ was used in combination with parallel imaging to reduce artifacts due to susceptibility changes at tissue interfaces around the spine, as previously reported. A finger pulse oximeter was used for cardiac triggering. The 
following parameters were used: $\mathrm{TR} / \mathrm{TE}=4500 / 66 \mathrm{~ms}$, in-plane acceleration (iPAT) $=2,30$ directions, $b$-value $650 \mathrm{~s} / \mathrm{mm}^{2}$, six $b=0$ volumes. Data were collected with reversed phase-encode blips (AP and PA), resulting in pairs of images with distortions going in opposite directions. From these pairs the susceptibility-induced off-resonance field was estimated and corrected using a method similar to that described in ${ }^{37}$. Subject motion and eddy-current induced distortions were corrected $^{38}$ as previously reported ${ }^{23}$.

\section{Spinal Cord Magnetic Resonance Spectroscopy}

Sagittal $\mathrm{T}_{2}$-weighted images similar to those acquired before spinal cord diffusion were used to position an $8 \times 6 \times 30 \mathrm{~mm}^{3}(1.44 \mathrm{ml})$ voxel in the spine, centered on the C4-C5 intervertebral disc. $\mathrm{B}_{0}$ shimming was performed using Siemens image-based shimming with a reduced field of view. Average water linewidth in the voxel was $13.0 \pm 3.4 \mathrm{~Hz}$ after shimming.

MR spectra were acquired using an optimized semi-LASER sequence with $\mathrm{TE}=28 \mathrm{~ms}, \mathrm{TR}=5 \mathrm{~s}$, and $2 \times 128$ averages. A detailed description of the sequence, including outer-volume suppression and VAPOR water suppression can be found in ${ }^{39}$. Individual shots were saved separately. A finger pulse oximeter was used for cardiac triggering. Unsuppressed water reference signals $(\mathrm{NT}=4)$ were collected for eddy-current correction, and to serve as an internal concentration reference for quantification. Water signal was also measured at different TEs $(28,35,50,70,100,200,300$, $500,800,1000,1500,3000$ and 4000ms) with one average per TE and $\mathrm{TR}=15 \mathrm{sec}$. The resulting $\mathrm{T}_{2}$ curve was fit with a bi-exponential function to determine the fraction of CSF and tissue in the voxel, assuming a $\mathrm{T}_{2}$ value of $740 \mathrm{~ms}$ for CSF. The entire procedure was repeated twice, yielding two spectra with 128 transients each, for a total of 256 transients.

Both individual shots and accumulating spectrum were monitored in real time for spectral quality by the operator. In case of movement (resulting in degraded water suppression, degraded water linewidth and/or lipid signal visible in single shots or in accumulated spectrum), the spectral acquisition was stopped (keeping the shots already acquired). Spine $\mathrm{T}_{2}$-weighted images were reacquired to reposition the voxel, adjust $\mathrm{B}_{0}$ shim again if necessary, and acquire the remaining shots. 


\section{MR Data Processing}

\section{Available Data}

In all, there were 88 visits (20 controls at baseline, 28 FRDA at baseline, 21 FRDA at 1 year, 19 FRDA at 2 years), and 3 datasets were acquired at each visit: Brain anatomical MRI (used for upper cervical spine morphometry), spinal cord dMRI and spinal cord MRS. Out of those 264 datasets, 12 datasets $(<5 \%)$ could not be used primarily because of excessive motion artifacts, but also artifacts due to braces, or missing data due to subject discomfort or anxiety. The final number of datasets used for the analysis was as follows: Baseline controls: $20 \mathrm{MRI}, 18 \mathrm{dMRI}, 19 \mathrm{MRS}$; Baseline FRDA: 26 MRI, 27 dMRI, 26 MRS; 1-year follow-up FRDA: 20 MRI, 20 dMRI, 20 MRS; 2-year follow-up FRDA: 19 MRI, 19 dMRI, 18 MRS.

\section{Anatomical MRI}

Brain $\mathrm{T}_{1}$-weighted images were analyzed using the Spinal Cord Toolbox (SCT) ${ }^{40}$ version 4.0 to extract spinal cord CSA and eccentricity at C1, C2 and C3 levels. The C2-C3 intervertebral disc was manually delineated to initialize the automatic labeling of vertebrae, after which deep learning algorithms in the SCT toolbox were used to segment the cord.

\section{Diffusion MRI}

The diffusion images were preprocessed to remove noise ${ }^{41}$ and Gibbs-ringing artefacts ${ }^{42}$. The images were then corrected for motion, as well as geometric and eddy-current distortions using FSL (topup and $e d d y$ ) ${ }^{38}$ and bias-field corrected. Subsequently, diffusion tensor fitting using robust estimation of tensors by outliers rejection ${ }^{43}$ was performed to estimate fractional anisotropy (FA), mean diffusivity (MD), radial diffusivity (RD) and axial diffusivity (AD) ${ }^{44}$. The mean $b=0$ image was segmented to aid with the registration of the PAM50 template ${ }^{45}$ into the native diffusion space of each participant. This registration step was initialized using the nonlinear deformation fields generated from the registration of the spine T2-weighted image to the PAM50 template during the segmentation of the spine T2-weighted image. Since it is difficult to identify the vertebral levels in the axially acquired diffusion image, the warping fields between the highcontrast spine T2-weighted image and the template were used to match vertebrae and thus 
indirectly and automatically label the vertebral levels in the diffusion image. Values for FA, MD, $\mathrm{RD}$ and $\mathrm{AD}$ within these spinal cord levels were extracted for subsequent statistical analysis.

\section{Magnetic Resonance Spectroscopy}

Individual shots in the first NT=128 acquisitions were inspected visually and any shot that had severely degraded water suppression, or showed lipid contamination, was removed. In most cases, SNR of metabolites on single shots was not sufficient for shot-to-shot frequency correction, therefore shots were averaged in blocks of 4 or 8 (depending on SNR), then frequency-aligned before summation. The procedure was repeated for the second NT=128 acquisition. The two resulting summed spectra were frequency-aligned and summed to yield a final single spectrum $(\mathrm{NT}=256)$. In some participants, one of the two spectra was either not acquired due to time constraints or not usable due to spectral quality, and only one NT=128 spectrum was used in the analysis instead of $\mathrm{NT}=256$.

Spectra were quantified using LCModel $6.3 \mathrm{H}^{46}$. The basis set comprised 18 metabolites: alanine, ascorbate, creatine (Cr), GABA, glucose, glutamine, glutamate, glycerophosphocholine (GPC), glutathione, myo-inositol, scyllo-inositol, lactate, phosphocreatine (PCr), phosphocholine (PCho), phenylethanolamine, $\mathrm{N}$-acetyl-aspartate (NAA), N-acetyl-aspartyl-glutamate (NAAG), taurine as well as an experimentally-measured metabolite-nulled macromolecule spectrum. Metabolites that cannot be distinguished at 3 Tesla are reported as a sum: total $\mathrm{N}$-acetyl-aspartate (tNAA) $=$ NAA $+\mathrm{NAAG}$, total creatine $(\mathrm{tCr})=\mathrm{Cr}+\mathrm{PCr}$, total choline $(\mathrm{tCho})=$ PCho + GPC. Based on CramerRao Lower Bounds (CRLB), only 4 metabolites were quantified reliably in the spine (CRLB < 20\%): tNAA, tCho, tCr and mIns.

\section{Effect of Scanner Upgrade}

The Trio scanner was upgraded to Prisma in the middle of the study. Out of 21 participants who returned for follow-up, 9 participants were scanned on Trio at baseline and 1-year follow-up, and on Prisma at 2-year follow-up (noted “TTP”), 2 participants were only scanned on Trio (“TT") (no 2-year follow-up), 7 participants were scanned on Trio at baseline and Prisma thereafter (“TPP”) 
and 3 participants were scanned only on Prisma ("PPP”). Scanning parameters were matched as closely as possible on Trio and Prisma.

To measure any potential bias due to this scanner upgrade, we scanned 5 additional subjects (not part of the $n=20$ control participants) on Trio before the upgrade, and on Prisma after the upgrade (see Suppl. Table 1). All measured parameters remained stable before and after the upgrade, with one exception: all diffusivity metrics (AD, RD and MD) were on average $25 \%$ higher on Prisma than on Trio. Therefore, in order to mitigate this effect, all underestimated values obtained on Trio were corrected (multiplied) by a factor of 1.25. The reason for this difference was traced to a single mismatched parameter (coil combination mode) set to "Sum of Squares" on Trio, and "Adaptive Combine" on Prisma ("Adaptative Combine" did not exist in VD13D). This resulted in different noise distributions on the two systems ${ }^{47}$ and affected diffusivity estimates (but not FA). Briefly, the Trio's "Sum of Squares" method leads to a diffusion signal that follows a noncentral- $\chi^{2}$ distribution with an elevated noise floor which artificially increase the diffusion signal, thereby underestimating diffusivity measures. Since we did not keep the raw per-coil data (so-called "TWIX" data), we were unable to reconstruct the Trio data with the "Adaptive Combine" method.

Following this correction of diffusivity values, we performed two analyses of the longitudinal data (which would be the most sensitive to any effect of the scanner upgrade) to rule out any remaining bias: One with all the data, and a second one with "same scanner only" data: For each participant, we kept only time points from the same scanner, i.e. for "TTP" we kept only "TT", for "TPP" we kept only "PP" and for "PPP" we kept "PPP". The "same scanner only" longitudinal results were then compared to those obtained with all data (see Suppl. Table 2). We found that longitudinal results were consistent between the two analyses, confirming that longitudinal results from all data were not significantly affected by the scanner upgrade.

\section{Statistical Analysis}

Missing data were left blank. To reduce the extent of multiple testing adjustment, the following metrics were chosen a priori (outcome measures): 
Morphometry: CSA and Eccentricity at C2-C3 obtained from SCT. The average of C2-C3 was chosen to allow comparison with manual segmentation performed at C2-C3 (Suppl. Figure 1). Diffusion: FA, MD, AD, RD averaged over C3-C6 (C2 and C7 were excluded because of inconsistent B0 shimming and image quality at the edge of the field of view).

Spectroscopy: Water-referenced concentrations in tNAA, mIns, $\mathrm{tCr}$, tCho, and ratio tNAA/mIns. The ratio tNAA/mIns was chosen because it combines the changes in tNAA (decrease) and mIns (increase) that is observed in many neurodegenerative diseases, maximizing the effect size. For longitudinal slopes and associations between clinical metrics and MR metrics, only the ratio tNAA/mIns was chosen due to higher variability observed in water-referenced concentrations. Clinical metrics: FARS total neuro, SARA, ADL, Functional staging, 9HPT non dominant.

MR metrics in patient and control groups were compared at baseline using linear models with group as the primary variable of interest, and age and sex as covariates.

Among FRDA participants only, associations of clinical metrics (response variables) with MR metrics (predictor variables), using data from all 3 visits, were estimated using linear mixed models, with subjects as random effects.

For each MR metric and each clinical metric, within-participant longitudinal slopes across all visits were estimated using linear regressions. These slopes were tested using one-sided one sample ttests. For each variable, we hypothesized that longitudinal changes would go in the same direction as cross-sectional differences at baseline. For example, if CSA was lower in FRDA participants than in controls at baseline, we hypothesized that CSA in FRDA participants would continue to decrease over time (null hypothesis: slope $<0)$. The sample size of the longitudinal cohort $(\mathrm{n}=21)$ was sufficient to detect slopes $>0$ (or $<0$ ) with an effect size of 0.5 (or -0.5 ) or better, with $\alpha=0.05$ and $\beta=0.7$.

For analyses that were repeated for multiple metrics, reported $p$-values were corrected for type-I error inflation due to multiple testing using the Holm-Bonferroni procedure across metrics within each MR modality (Morphometry: 2 metrics, Diffusion: 4 metrics, Spectroscopy: 5 metrics at baseline, 1 metric longitudinally; Clinical: 5 metrics). 


\section{DATA AVAILABILITY}

The data that support the findings of this study are available upon reasonable request from the corresponding author. The data are not publicly available due to their containing information that could compromise the privacy of research participants.

\section{RESULTS}

\section{Cross-sectional Cohort Findings at Baseline}

\section{Morphometry}

Spinal cord CSA at C2-C3 was 31\% lower $\left(\mathrm{p}<4.10^{-17}\right)$ and eccentricity was $10 \%$ higher $\left(\mathrm{p}<5.10^{-}\right.$ ${ }^{7}$ ) in FRDA participants relative to controls (Figure 1 and Table 2). There was no overlap in CSA values between the FRDA and control groups.

\section{Diffusion}

FA was $24 \%$ lower $\left(p=10^{-9}\right)$ in FRDA participants relative to controls (Figure 3). All three diffusivity values were higher in FRDA participants, with RD 56\% higher $\left(\mathrm{p}=2.10^{-9}\right)$, MD 35\% higher $\left(\mathrm{p}=10^{-8}\right)$ and AD $17 \%$ higher $\left(\mathrm{p}=4.10^{-5}\right)$ than in controls (Table 2$)$.

\section{Spectroscopy}

In spite of the small volume, the main resonances (tCho, tCr, tNAA, mIns) were clearly visible in MR spectra (Figure 2). The average water linewidth was $13.0 \pm 3.4 \mathrm{~Hz}$, average metabolite linewidth returned by LCModel was $15.4 \pm 3.8 \mathrm{~Hz}$ and average signal-to-noise ratio (SNR, with noise defined as $2 * \mathrm{RMS}_{\text {noise }}$ ) returned by LCModel was $5.0 \pm 1.6$.

The spectral pattern was markedly different in the FRDA participants relative to controls, with a lower tNAA peak at $2.01 \mathrm{ppm}$ and a higher mIns peak at $3.55 \mathrm{ppm}$ (Figure 3 ). The concentration of tNAA was $36 \%$ lower $\left(\mathrm{p}<6.10^{-9}\right)$ and the concentration of mIns was $37 \%$ higher $\left(\mathrm{p}<2.10^{-6}\right)$ in FRDA participants than in controls (Table 2). As a result, the ratio $\mathrm{tNAA} / \mathrm{mIns}$ was $52 \%$ lower in 
FRDA participants compared to controls $\left(\mathrm{p}<2.10^{-13}\right)$. There was almost no overlap in $\mathrm{tNAA} / \mathrm{mIns}$ values between the two groups. All patients had a tNAA $/ \mathrm{mIns}$ ratio below 0.85 , while all controls had a tNAA/mIns ratio higher than 0.85 , with one exception: a 10 y.o. control participant had a tNAA/mIns ratio of 0.6 .

All cross-sectional results, as well as cross-sectional effect sizes (Cohen's d), are summarized in Table 2.

\section{Longitudinal Cohort Findings}

Annual rates of change (slopes) for each MR metric and each clinical metric are summarized in Table 3. Effect size is reported as Standardized Response Mean (SRM) defined as mean(slope)/SD(slope).

\section{Morphometry}

Spinal cord morphometry at C2-C3 using SCT showed a mean rate of atrophy of $-2.4 \%$ per year $(\mathrm{SRM}=-0.95)$, with no significant change in eccentricity (Figure 1). These findings were broadly consistent across individual vertebral levels C1, C2 and C3, with the largest effect size (-1.23) for the average C1-C2 (Suppl. Table 3). The effect size was smaller for C3 (-0.78) than for C1 or C2 (-1.13 and -1.07 respectively) due to higher standard deviation at $\mathrm{C} 3$, possibly reflecting the decreasing coil sensitivity and lower SNR at the edge of brain T1 images.

In a separate analysis, spinal cord area and eccentricity were also obtained through manual segmentation of the spinal cord at C2-C3 using SpineSeg ${ }^{48}$ by three independent raters in a blind fashion (see Suppl. Figure 1). The rate of atrophy from the average CSA across the three raters ($2.9 \%$ ) was slightly higher than the one found using the automated method SCT $(-2.4 \%)$ and had slightly lower SRM (-0.87 vs -0.95). All three individual raters found a decrease in CSA over time, but with lower SRM (ranging from -0.62 to -0.79), suggesting that manual segmentation introduces more variability than SCT (Suppl. Figure 1).

\section{Diffusion}


Fractional anisotropy averaged across C3-C6 showed a trend (raw p $<0.02$, corrected $\mathrm{p}<0.08$ ) in yearly decrease of $-3.2 \%$ with a Cohen's $d$ of -0.50 . There was no significant change for any of the diffusivity metrics (MD, RD, AD) averaged across C3-C6. In post-hoc analyses (Suppl. Table 3), FA values appeared to be most reliable (lower standard deviation and greater effect size) at the center of the imaging volume $(\mathrm{C} 4-\mathrm{C} 5)$ were $\mathrm{B}_{0}$ shimming is optimal and image quality is higher. FA values averaged between C4 and C5 confirmed the trend in yearly decrease with a change of $5.6 \%$ (raw $\mathrm{p}<0.0002$ ) and Cohen's $\mathrm{d}$ of -1.0 .

\section{Spectroscopy}

tNAA $/$ mIns showed a significant $(\mathrm{p}<0.03$ ) yearly decrease of $-5.8 \%$ with a SRM of -0.51 . Because individual metabolite concentrations had higher variability than the ratio tNAA/mIns, slopes for individual metabolites were not included in the main analysis, but are provided in Suppl. Table 3 for information. While none of the individual metabolites showed a statistically significant change over time, numerical results suggest that the longitudinal decrease in tNAA/mIns may be driven both by a decrease in tNAA (-3.9\% / year) and an increase in mIns ( $+0.7 \%$ / year). This is supported by the fact that the ratio $\mathrm{tNAA} / \mathrm{tCr}$ decreased and the ratio $\mathrm{mIns} / \mathrm{tCr}$ increased, while $\mathrm{tCr}$ was relatively stable (Suppl. Table 3).

For comparison, clinical assessments showed an average increase of 2.0 points/year in SARA score $(\mathrm{SRM}=1.89), 5.3$ points/year in FARS total neurological subscale $(\mathrm{SRM}=1.44), 2.0$ points/year in ADL $(\mathrm{SRM}=1.4)$ and 0.4 point/year in functional staging $(\mathrm{SRM}=1.49)$. The nine-hole peg test (non-dominant hand) showed an average increase of 3.4 seconds per year with an SRM of 0.86 .

\section{Correlations of MR metrics with Clinical Scales}

Many of the measured MR parameters correlated with clinical parameters (Table 4). The strongest effects were observed for the spinal cord CSA, followed by FA and tNAA/mIns. CSA correlated negatively with all clinical parameters: FARS, SARA, ADL, functional staging and 9HPT. For example, CSA correlated negatively with FARS total neuro with $\mathrm{R}=-0.60$ and $\mathrm{p}=4.10^{-7}$ (Figure 4). FA correlated negatively with FARS total neuro, functional staging and ADL, while tNAA/mIns negatively correlated with functional staging, ADL and 9HPT non-dominant. 


\section{DISCUSSION}

To our knowledge, these are the first quantitative longitudinal MR results ever reported in the spinal cord in FRDA. Our data demonstrate the ability to monitor disease progression noninvasively in the spinal cord in FRDA, which is critical to assess how potential treatments affect the nervous system in upcoming clinical trials.

In addition, while a handful of studies have reported cross-sectional results in the spinal cord in FRDA with morphometry, and one recent study with DTI but none with MRS, our study provides unique multimodal cross-sectional data. The multimodal approach allowed assessment of anatomy, microstructure and neurochemistry in the same subjects, providing different and complementary measures of neurodegeneration.

Lastly, use of an early-stage cohort (as we did here, with only 5.5 years from onset and 2.3 years from diagnosis) will be crucial for planning of treatment development and testing going forward because efficacy of potential treatments would presumably be maximal if administered in the early stage of the disease, and because the faster disease progression at early stage would make it easier to detect an effect of potential treatments.

All three MR modalities (anatomical MRI, dMRI and MRS) showed large cross-sectional alterations in FRDA participants relative to controls, even at such an early stage. In addition, several MR metrics showed sensitivity to disease progression over time, with spinal cord CSA showing the largest longitudinal effect size among all MR metrics.

\section{Morphometry}

The smaller spinal cord area and higher eccentricity in FRDA compared to controls is consistent with previously reported results using MRI 11,12,15-17, as well as post-mortem pathology results ${ }^{49}$. While these cohorts are not directly comparable, results are remarkably consistent between studies, 
and show that the upper cervical spinal cord is generally $\sim 30-40 \%$ smaller in FRDA relative to controls, and eccentricity is $\sim 10 \%$ higher.

\section{Diffusion MRI}

Our dMRI cross-sectional findings are consistent with a recent study that reported lower FA and higher diffusivity in total white matter and in specific white matter tracts in FRDA ${ }^{30}$. Both studies found that FA in total white matter across C3-C6 (C2-C5 in ${ }^{30}$ ) was about 25\% lower in FRDA participants compared to controls, albeit Hernandez et al. ${ }^{30}$ reported a smaller cross-sectional effect size (Cohen's d) of about -0.85 (compared to -2.5 here), which could be due to the larger range of disease severity in their cohort (IQR for FARS total neuro $=41$ vs 14.8 in the present study) and therefore larger SD, or due to differences in MR acquisition parameters affecting the SNR of images.

Both studies also found that RD in total white matter was much higher in FRDA than in controls $\left(+56 \%\right.$ in our study, about $+160 \%$ in $\left.{ }^{30}\right)$, while the increase in AD was smaller $(+17 \%$ in our study, $+6 \%$ in ${ }^{30}$ )

The lower FA and higher diffusivity observed in the spinal cord are similar to those observed in the CNS in many neurodegenerative diseases and are consistent with neurodegeneration.

\section{Magnetic Resonance Spectroscopy}

Higher tNAA and lower mIns have been observed in numerous neurodegenerative brain disorders and are not specific to FRDA. However, alterations observed in the spinal cord in the present study were surprisingly large, and much larger than those observed in the brain in FRDA ${ }^{50}$. In fact, the differences are so pronounced that disease status can practically be determined from visual inspection of the spectra alone.

Myo-inositol is often associated with glia and myelination, while tNAA, synthesized only in neurons, is considered a neuronal marker. Therefore, our findings suggest both neuronal loss and abnormal myelination.

\section{Longitudinal Effect sizes}

Among MR metrics, the largest longitudinal effect size (SRM) was found for cross-sectional area, with an effect size of -0.95 at C2-C3. Post-hoc analyses showed that SRM was even higher for 
medRxiv preprint doi: https://doi.org/10.1101/2022.01.28.22270048; this version posted January 30, 2022. The copyright holder for this preprint (which was not certified by peer review) is the author/funder, who has granted medRxiv a license to display the preprint in perpetuity. It is made available under a CC-BY-NC-ND 4.0 International license .

CSA at C1-C2 (-1.23) (Suppl. Table 3). The lower effect size at C3 (-0.78) could be due to lower SNR at the edge of the field of view in the brain T1-weighted image. Studies with larger spinal cord coverage would be needed to investigate longitudinal changes in lower cervical and thoracic areas. However, we hypothesize that $\mathrm{C} 1-\mathrm{C} 2$ will provide close to maximum SRM because that region contains the highest number of ascending axons, and therefore would presumably undergo the largest absolute decrease in volume from neurodegeneration.

The longitudinal decrease in FA averaged over C3-C6 was borderline significant after correction for multiple testing, but post-hoc analysis suggests that FA averaged over C4-C5 could be more precise. Indeed, when looking at individual levels (Suppl. Table 3), the SD on diffusion parameters was consistently lowest at C4-C5 and highest at C2 and C7. This suggests that measurement precision is highest in the center of the dMRI field of view, where shim quality is highest.

A tract-specific analysis using SCT, similar to what was recently reported in ${ }^{30}$, did not reveal improved longitudinal effect size (SRM) in the dorsal columns and corticospinal tract (Suppl. Table 5).

For MRS, the ratio tNAA/mIns was by far the most sensitive longitudinal metric as expected. Other ratios such as $\mathrm{tNAA} / \mathrm{tCr}$ and $\mathrm{mIns} / \mathrm{tCr}$ were less sensitive to disease progression, but suggest that both a decrease in tNAA and an increase in mIns contribute to the longitudinal decrease in tNAA/mIns (Suppl. Table 3).

Overall, MR metrics had lower effect sizes than clinical metrics, with spinal cord cross-sectional area coming the closest. However, now knowing where to look, we expect that MR acquisition can be further optimized to improve the effect size of MR metrics. For example, spinal cord CSA was determined here using brain T1 images with $1 \mathrm{~mm}$ isotropic resolution and suboptimal SNR below C3 cervical level. Future studies could easily use $0.8 \mathrm{~mm}$ isotropic, and improved coverage of the cervical cord by activating corresponding coil elements in the 64-channel spine+neck receive coil.

We also noted that SRMs for clinical metrics in the present study appear higher than those reported in literature ${ }^{51}$. For example, one study reported an increase in FARS total neuro of $4.1 \pm 7.84$ (Table 10 in ${ }^{51}$ ) in a young cohort (BL age < 16) after one year, corresponding to an SRM of 0.52. In the present study, the annual increase in FARS total neuro (from 1-year data only) was $5.2 \pm$ 4.5 corresponding to an SRM of 1.14 (Suppl. Table 4), even though the cohort was older (BL age 
=19). It appears that the higher SRM in our study is primarily due to lower SD (4.5 vs 7.84), which could be explained by the fact that all clinical assessments were performed by a single rater (no inter-rater variability).

\section{True Annual Effect Size}

Effect sizes (SRMs) reported in Table 3 are for annual slopes obtained from 2-year data (i.e., 3 time points per participant). To plan clinical trials, it would also be useful to know the "true" annual SRM, namely SRM size obtained from 1-year data only (i.e., 2 time points per participant). These SRMs are reported in Suppl. Table 4. As expected, true annual SRMs were lower than annual SRMs obtained from 2-year data. For example, the effect size for CSA at C2-C3 was -0.95 for annual slope from 2-year data, and -0.71 for annual slopes from 1-year data only.

\section{Hypoplasia vs. Neurodegeneration}

The large differences observed in FRDA participants relative to controls (for example, $-52 \%$ lower tNAA/mIns) suggest that alterations may already be present well before onset. This is consistent with the hypothesis that alterations in the spinal cord are largely due to hypoplasia rather than neurodegeneration ${ }^{49}$. While previously reported correlations between CSA and measures of disease severity at baseline suggested that neurodegeneration was also present in FRDA ${ }^{15}$, our longitudinal data provides direct evidence and quantifies this neurodegeneration, with a rate of atrophy of $-2.4 \%$ per year in our early-stage cohort.

\section{MR Metrics for Monitoring Disease Progression in Premanifest Individuals}

While CSA was the MR metric with the strongest longitudinal effect size in our cohort, other MR metrics could also play a key role, especially during the pre-manifest phase. For example, it is possible that neurochemical (MRS) and microstructural (DTI) alterations occur earlier during development than alterations in cross-sectional area (morphometry). While identification of premanifest individuals with FRDA is currently rare, perinatal genetic testing will likely become more widespread in the near future once the first effective treatments become available. MR modalities capable of detecting changes during the premanifest phase could therefore play a crucial role in assessing treatment efficacy during that phase, and in determining how early to start treatment. 


\section{Possible Improvements to the MR protocol}

With results now providing a better picture of the expected cross-sectional alterations and longitudinal changes in the spinal cord in FRDA, the MR protocol could be streamlined and shortened substantially, benefitting from recent advances in hardware and pulse sequences. For example, while spine dMRI acquisition lasted about 40min in the present study, it can be reduced to $\sim 4 \min ^{21}$. Similarly, the length of spinal cord MRS was about $40 \mathrm{~min}$ (including all adjustments and shimming). Shorter TR, fewer shots (due to improved SNR with available 64ch head-neck coils), faster shimming using FASTMAP, and improved workflow through automation of adjustment steps now allow acquisition of similar data in about $10 \mathrm{~min}$.

\section{Limitations of the Study}

Our study has a number of limitations. First, follow-up scans were only performed in FRDA participants, and not in controls. Therefore, slopes of change over time in FRDA were tested compared to zero, assuming that control values remain stable during the follow-up period. Indeed, published studies suggest that longitudinal changes in control participants are unlikely to explain the changes observed in our FRDA participants (average age 19-20 years, range 10-35 years). For example, large cross-sectional studies in healthy participants across age have shown that CSA increases from age 10 to early-mid-twenties, and remains stable from $20 \mathrm{~s}$ to $30 \mathrm{~s}{ }^{52,53}$. Therefore, we would not expect to see a decrease in CSA in controls in our cohort. For spine MRS, concentrations reported in healthy controls show less than $1 \%$ decrease in tNAA and no change in mIns across ages in the 20-70 age range ${ }^{54}$. For dMRI, FA and MD have been shown to be fairly stable, with less than $0.2 \%$ change per year in the $10-40$ age range ${ }^{55}$. Therefore, it seems unlikely that the observed decrease in CSA, tNAA/mIns and DTI metrics in the present study would be due to normal aging.

Another limitation is that the scanner was upgraded midway through the study. However, the testretest data obtained before and after upgrade, and the fact that we find similar results with "same scanner" data and with "all data" gives us high confidence that the longitudinal changes reported here were not substantially affected by the scanner upgrade. 


\section{Need for Larger, Multi-Site Study}

Finally, while our single-site study provides the first quantitative longitudinal results in the spinal cord in FRDA, they were obtained in an early-stage cohort and cannot be generalized to later disease stages. Natural history studies of clinical scales have shown that disease progression in FRDA is faster at early stage ${ }^{51}$, as well in younger patients and in classical onset FRDA as opposed to late onset ${ }^{56}$. Our cohort in the present study included only classical onset FRDA participants, most of whom were young and early stage. The average annual increase (slopes from 2-year data) was $5.3 \pm 3.7$ for FARS total neuro, $2.0 \pm 1.1$ for SARA and $2.0 \pm 1.4$ for ADL (Table 3). Based on natural studies of clinical scales, changes in MR metrics are likely slower in older cohorts, late onset cohorts and/or non-ambulatory cohorts.

Larger multi-site studies are needed to cover the entire range of disease severity and duration and gain a fuller picture of disease progression in the spinal cord in FRDA. A new multi-site longitudinal neuroimaging study (TRACK-FA ${ }^{1}$ ) has now started and aims to scan 200 patients and $>100$ controls at 3 time points over 24 months. This international effort, involving seven sites on four continents, will use an improved and streamlined neuroimaging protocol that includes the modalities presented in this paper.

\section{FUNDING}

This work was supported by primarily by the Friedreich's Ataxia Research Alliance (FARA), with additional support from Ataxia UK, GoFAR, the CureFA Foundation and the Bob Allison Ataxia Research Center (BAARC). Aside from funding, FARA also assisted with recruitment of FRDA participants. The Center for Magnetic Resonance Research is supported by NIH grants P41EB027061 and P30NS076408. The 3 Tesla scanner upgrade was funded in part by NIH instrumentation grant 1S10OD017974.

\section{COMPETING INTERESTS}

\footnotetext{
${ }^{1} \mathrm{https}: / /$ www.monash.edu/medicine/trackfa
} 
medRxiv preprint doi: https://doi.org/10.1101/2022.01.28.22270048; this version posted January 30, 2022. The copyright holder for this preprint (which was not certified by peer review) is the author/funder, who has granted medRxiv a license to display the preprint in perpetuity. It is made available under a CC-BY-NC-ND 4.0 International license.

C.L. and P.G.H. received research grants from Minoryx Therapeutics. All other authors stated that they have no conflicts of interest to report. 


\section{REFERENCES}

1. Cook A, Giunti P. Friedreich's ataxia: clinical features, pathogenesis and management. British medical bulletin. Dec 1 2017;124(1):19-30. doi:10.1093/bmb/ldx034

2. Vankan P. Prevalence gradients of Friedreich's ataxia and R1b haplotype in Europe co-localize, suggesting a common Palaeolithic origin in the Franco-Cantabrian ice age refuge. J Neurochem. Aug 2013;126 Suppl 1:11-20. doi:10.1111/jnc.12215

3. Campuzano V, Montermini L, Molto MD, et al. Friedreich's ataxia: autosomal recessive disease caused by an intronic GAA triplet repeat expansion. Science. Mar 8 1996;271(5254):1423-7. doi:10.1126/science.271.5254.1423

4. Durr A, Cossee M, Agid Y, et al. Clinical and genetic abnormalities in patients with Friedreich's ataxia. $N$ Engl J Med. Oct 17 1996;335(16):1169-75. doi:10.1056/NEJM199610173351601

5. $\quad$ Pandolfo M. Friedreich ataxia. Arch Neurol. Oct 2008;65(10):1296-303. doi:10.1001/archneur.65.10.1296

6. Delatycki MB, Williamson R, Forrest SM. Friedreich ataxia: an overview. Journal of medical genetics. Jan 2000;37(1):1-8. doi:10.1136/jmg.37.1.1

7. Koeppen AH. Nikolaus Friedreich and degenerative atrophy of the dorsal columns of the spinal cord. J Neurochem. 2013;126 Suppl 1(0 1):4-10. doi:10.1111/jnc.12218

8. Wessell K, Schroth G, Diener HC, Müller-Forell W, Dichgans J. Significance of MRI-confirmed atrophy of the cranial spinal cord in Friedreich's ataxia. journal article. European archives of psychiatry and neurological sciences. May 01 1989;238(4):225-230. doi:10.1007/bf00381470

9. Wullner U, Klockgether T, Petersen D, Naegele T, Dichgans J. Magnetic resonance imaging in hereditary and idiopathic ataxia. Neurology. Feb 1993;43(2):318-25. doi:10.1212/wnl.43.2.318

10. De Michele G, Di Salle F, Filla A, et al. Magnetic resonance imaging in "typical" and "late onset" Friedreich's disease and early onset cerebellar ataxia with retained tendon reflexes. Ital J Neurol Sci. Jun 1995;16(5):303-8.

11. Klockgether T, Petersen D, Grodd W, Dichgans J. Early onset cerebellar ataxia with retained tendon reflexes. Clinical, electrophysiological and MRI observations in comparison with Friedreich's ataxia. Brain. Aug 1991;114 ( Pt 4):1559-73.

12. Mascalchi M, Salvi F, Piacentini S, Bartolozzi C. Friedreich's ataxia: MR findings involving the cervical portion of the spinal cord. AJR Am J Roentgenol. Jul 1994;163(1):187-91. doi:10.2214/ajr.163.1.8010211

13. Koeppen AH, Davis AN, Morral JA. The cerebellar component of Friedreich's ataxia. Acta Neuropathol. Sep 2011;122(3):323-30. doi:10.1007/s00401-011-0844-9

14. Koeppen AH, Morral JA, Davis AN, et al. The dorsal root ganglion in Friedreich's ataxia. Acta Neuropathol. Dec 2009;118(6):763-76. doi:10.1007/s00401-009-0589-x

15. Rezende TJR, Martinez ARM, Faber I, et al. Developmental and neurodegenerative damage in Friedreich's ataxia. Eur J Neurol. Mar 2019;26(3):483-489. doi:10.1111/ene.13843

16. Chevis CF, da Silva CB, D'Abreu A, et al. Spinal cord atrophy correlates with disability in Friedreich's ataxia. Cerebellum. Feb 2013;12(1):43-7. doi:10.1007/s12311-012-0390-6 
17. Dogan I, Romanzetti S, Didszun C, et al. Structural characteristics of the central nervous system in Friedreich ataxia: an in vivo spinal cord and brain MRI study. $J$ Neurol Neurosurg Psychiatry. May 2019;90(5):615-617. doi:10.1136/jnnp-2018-318422

18. Basser PJ, Jones DK. Diffusion-tensor MRI: theory, experimental design and data analysis - a technical review. NMR Biomed. Nov-Dec 2002;15(7-8):456-67. doi:10.1002/nbm.783

19. O'Donnell LJ, Daducci A, Wassermann D, Lenglet C. Advances in computational and statistical diffusion MRI. NMR Biomed. Apr 2019;32(4):e3805. doi:10.1002/nbm.3805

20. Cohen-Adad J, Alonso-Ortiz E, Abramovic M, et al. Open-access quantitative MRI data of the spinal cord and reproducibility across participants, sites and manufacturers. Sci Data. Aug 16 2021;8(1):219. doi:10.1038/s41597-021-00941-8

21. Cohen-Adad J, Alonso-Ortiz E, Abramovic M, et al. Generic acquisition protocol for quantitative MRI of the spinal cord. Nat Protoc. Oct 2021;16(10):4611-4632. doi:10.1038/s41596-021-00588-0

22. Labounek R, Valosek J, Horak T, et al. HARDI-ZOOMit protocol improves specificity to microstructural changes in presymptomatic myelopathy. Sci Rep. Oct 16 2020;10(1):17529. doi:10.1038/s41598-020-70297-3

23. Pisharady PK, Eberly LE, Cheong I, et al. Tract-specific analysis improves sensitivity of spinal cord diffusion MRI to cross-sectional and longitudinal changes in amyotrophic lateral sclerosis. Commun Biol. Jul 10 2020;3(1):370. doi:10.1038/s42003-020-1093-Z

24. Martin Noguerol T, Barousse R, Amrhein TJ, Royuela-Del-Val J, Montesinos P, Luna A. Optimizing Diffusion-Tensor Imaging Acquisition for Spinal Cord Assessment: Physical Basis and Technical Adjustments. Radiographics. Mar-Apr 2020;40(2):403-427. doi:10.1148/rg.2020190058

25. Xu J, Shimony JS, Klawiter EC, et al. Improved in vivo diffusion tensor imaging of human cervical spinal cord. Neuroimage. Feb 15 2013;67:64-76. doi:10.1016/j.neuroimage.2012.11.014

26. Holder CA, Muthupillai R, Mukundan S, Jr., Eastwood JD, Hudgins PA. Diffusion-weighted MR imaging of the normal human spinal cord in vivo. AJNR Am J Neuroradiol. Nov-Dec 2000;21(10):1799806.

27. Valosek J, Labounek R, Horak T, et al. Diffusion magnetic resonance imaging reveals tractspecific microstructural correlates of electrophysiological impairments in non-myelopathic and myelopathic spinal cord compression. Eur J Neurol. Nov 2021;28(11):3784-3797. doi:10.1111/ene.15027

28. Wolanczyk M, Bladowska J, Koltowska A, et al. Diffusion tensor imaging of normal-appearing cervical spinal cords in patients with multiple sclerosis: Correlations with clinical evaluation and cerebral diffusion tensor imaging changes. Preliminary experience. Adv Clin Exp Med. Apr 2020;29(4):441-448. doi:10.17219/acem/116754

29. Freund P, Seif M, Weiskopf N, et al. MRI in traumatic spinal cord injury: from clinical assessment to neuroimaging biomarkers. Lancet Neurol. Dec 2019;18(12):1123-1135. doi:10.1016/S1474-4422(19)30138-3

30. Hernandez A, Rezende TJR, Martinez ARM, de Brito MR, Franca MC, Jr. Tract-Specific Spinal Cord Diffusion Tensor Imaging in Friedreich's Ataxia. Mov Disord. Oct 29 2021; doi:10.1002/mds.28841

31. Wyss PO, Hock A, Kollias S. The Application of Human Spinal Cord Magnetic Resonance Spectroscopy to Clinical Studies: A Review. Semin Ultrasound CT MR. Apr 2017;38(2):153-162. doi: $10.1053 /$ j.sult.2016.07.005 
32. Hock A, Fuchs A, Boesiger P, Kollias SS, Henning A. Electrocardiogram-triggered, higher order, projection-based $\mathrm{B}(0)$ shimming allows for fast and reproducible shim convergence in spinal cord (1)H MRS. NMR Biomed. Mar 2013;26(3):329-35. doi:10.1002/nbm.2852

33. Cooke FJ, Blamire AM, Manners DN, Styles P, Rajagopalan B. Quantitative proton magnetic resonance spectroscopy of the cervical spinal cord. Magn Reson Med. Jun 2004;51(6):1122-8.

doi: $10.1002 / \mathrm{mrm} .20084$

34. Gomez-Anson B, MacManus DG, Parker GJ, et al. In vivo 1H-magnetic resonance spectroscopy of the spinal cord in humans. Neuroradiology. Jul 2000;42(7):515-7.

35. Subramony SH, May W, Lynch D, et al. Measuring Friedreich ataxia: Interrater reliability of a neurologic rating scale. Neurology. Apr 12 2005;64(7):1261-2.

doi:10.1212/01.WNL.0000156802.15466.79

36. Porter DA, Heidemann RM. High resolution diffusion-weighted imaging using readoutsegmented echo-planar imaging, parallel imaging and a two-dimensional navigator-based reacquisition. Magn Reson Med. Aug 2009;62(2):468-75. doi:10.1002/mrm.22024

37. Andersson JL, Skare S, Ashburner J. How to correct susceptibility distortions in spin-echo echoplanar images: application to diffusion tensor imaging. Neuroimage. Oct 2003;20(2):870-88. doi:10.1016/S1053-8119(03)00336-7

38. Andersson JLR, Sotiropoulos SN. An integrated approach to correction for off-resonance effects and subject movement in diffusion MR imaging. Neuroimage. Jan 15 2016;125:1063-1078. doi:10.1016/j.neuroimage.2015.10.019

39. Oz G, Tkac I. Short-echo, single-shot, full-intensity proton magnetic resonance spectroscopy for neurochemical profiling at $4 \mathrm{~T}$ : validation in the cerebellum and brainstem. Magn Reson Med. Apr 2011;65(4):901-10. doi:10.1002/mrm.22708

40. De Leener B, Levy S, Dupont SM, et al. SCT: Spinal Cord Toolbox, an open-source software for processing spinal cord MRI data. Neuroimage. Jan 15 2017;145(Pt A):24-43. doi:10.1016/j.neuroimage.2016.10.009

41. Veraart J, Novikov DS, Christiaens D, Ades-Aron B, Sijbers J, Fieremans E. Denoising of diffusion MRI using random matrix theory. Neuroimage. Nov 15 2016;142:394-406. doi:10.1016/j.neuroimage.2016.08.016

42. Kellner E, Dhital B, Kiselev VG, Reisert M. Gibbs-ringing artifact removal based on local subvoxel-shifts. Magn Reson Med. Nov 2016;76(5):1574-1581. doi:10.1002/mrm.26054

43. Chang LC, Jones DK, Pierpaoli C. RESTORE: robust estimation of tensors by outlier rejection. Magn Reson Med. May 2005;53(5):1088-95. doi:10.1002/mrm.20426

44. Lenglet C. Diffusion Tensor Imaging. In: Toga AW, ed. Brain Mapping. Academic Press; 2015:245-251.

45. De Leener B, Fonov VS, Collins DL, Callot V, Stikov N, Cohen-Adad J. PAM50: Unbiased multimodal template of the brainstem and spinal cord aligned with the ICBM152 space. Neuroimage. Jan 15 2018;165:170-179. doi:10.1016/j.neuroimage.2017.10.041

46. Provencher SW. Estimation of metabolite concentrations from localized in vivo proton NMR spectra. Magn Reson Med. Dec 1993;30(6):672-9. 
medRxiv preprint doi: https://doi.org/10.1101/2022.01.28.22270048; this version posted January 30, 2022. The copyright holder for this preprint (which was not certified by peer review) is the author/funder, who has granted medRxiv a license to display the preprint in perpetuity.

It is made available under a CC-BY-NC-ND 4.0 International license .

47. Sotiropoulos SN, Moeller S, Jbabdi S, et al. Effects of image reconstruction on fiber orientation mapping from multichannel diffusion MRI: reducing the noise floor using SENSE. Magn Reson Med. Dec 2013;70(6):1682-9. doi:10.1002/mrm.24623

48. Bergo FPG. SpineSeg: A Tool for Measurement of Spinal Cord Atrophy. Accessed 22 Nov 2021, http://www.bergo.eng.br/academic/spineseg/index.php

49. Koeppen AH, Becker AB, Qian J, Feustel PJ. Friedreich Ataxia: Hypoplasia of Spinal Cord and Dorsal Root Ganglia. J Neuropathol Exp Neurol. Feb 1 2017;76(2):101-108. doi:10.1093/jnen/nlw111

50. Iltis I, Hutter D, Bushara KO, et al. (1)H MR spectroscopy in Friedreich's ataxia and ataxia with oculomotor apraxia type 2. Brain Res. Oct 28 2010;1358:200-10. doi:10.1016/j.brainres.2010.08.030

51. Patel M, Isaacs CJ, Seyer L, et al. Progression of Friedreich ataxia: quantitative characterization over 5 years. Ann Clin Transl Neurol. Sep 2016;3(9):684-94. doi:10.1002/acn3.332

52. Kato F, Yukawa Y, Suda K, Yamagata M, Ueta T. Normal morphology, age-related changes and abnormal findings of the cervical spine. Part II: Magnetic resonance imaging of over 1,200 asymptomatic subjects. Eur Spine J. Aug 2012;21(8):1499-507. doi:10.1007/s00586-012-2176-4

53. Ishikawa M, Matsumoto M, Fujimura Y, Chiba K, Toyama Y. Changes of cervical spinal cord and cervical spinal canal with age in asymptomatic subjects. Spinal Cord. Mar 2003;41(3):159-63. doi:10.1038/sj.sc.3101375

54. Abdel-Aziz K, Solanky BS, Yiannakas MC, et al. Age related changes in metabolite concentrations in the normal spinal cord. PLoS One. 2014;9(10):e105774.

doi:10.1371/journal.pone.0105774

55. Agosta F, Lagana M, Valsasina $\mathrm{P}$, et al. Evidence for cervical cord tissue disorganisation with aging by diffusion tensor MRI. Neuroimage. Jul 1 2007;36(3):728-35.

doi:10.1016/j.neuroimage.2007.03.048

56. Reetz K, Dogan I, Hilgers RD, et al. Progression characteristics of the European Friedreich's Ataxia Consortium for Translational Studies (EFACTS): a 4-year cohort study. Lancet Neurol. May 2021;20(5):362-372. doi:10.1016/S1474-4422(21)00027-2 
medRxiv preprint doi: https://doi.org/10.1101/2022.01.28.22270048; this version posted January 30, 2022. The copyright holder for this preprint (which was not certified by peer review) is the author/funder, who has granted medRxiv a license to display the preprint in perpetuity.

It is made available under a CC-BY-NC-ND 4.0 International license .

\section{FIGURES}
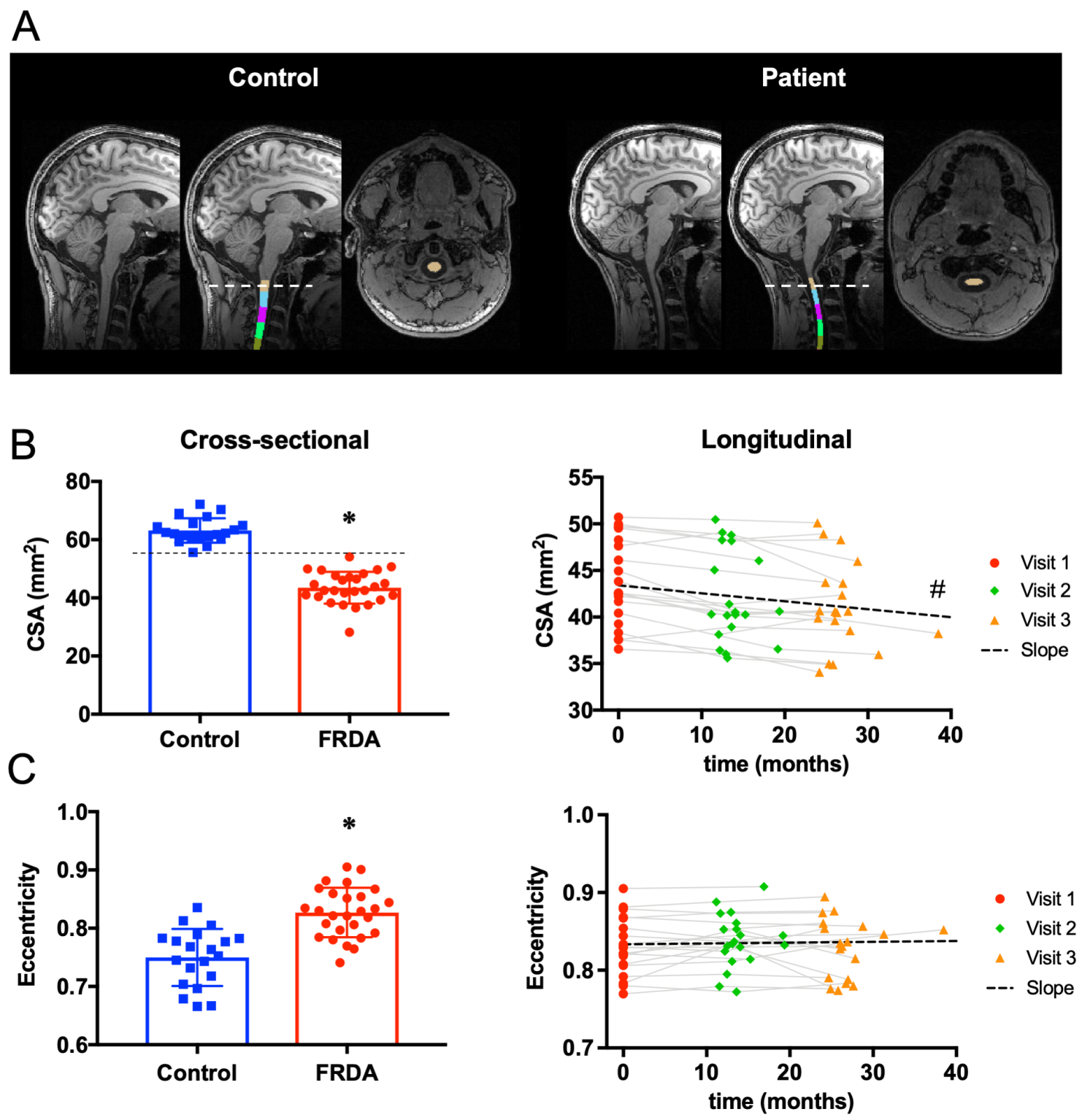

Figure 1. Spinal cord morphometry. (A) Brain T1 images showing automated segmentation of the upper cervical spinal cord using SCT. (B) Left: Cross-sectional comparison of cross-sectional area at C2-C3 in FRDA vs. Control at baseline. Right: Longitudinal change in cross-sectional area at C2-C3 in FRDA. (C) Left: Cross-sectional comparison of eccentricity at C2-C3 in FRDA vs. Control at baseline. Right: Longitudinal change in eccentricity at C2-C3 in FRDA. * FRDA statistically different from control, \# slope statistically different from zero. 

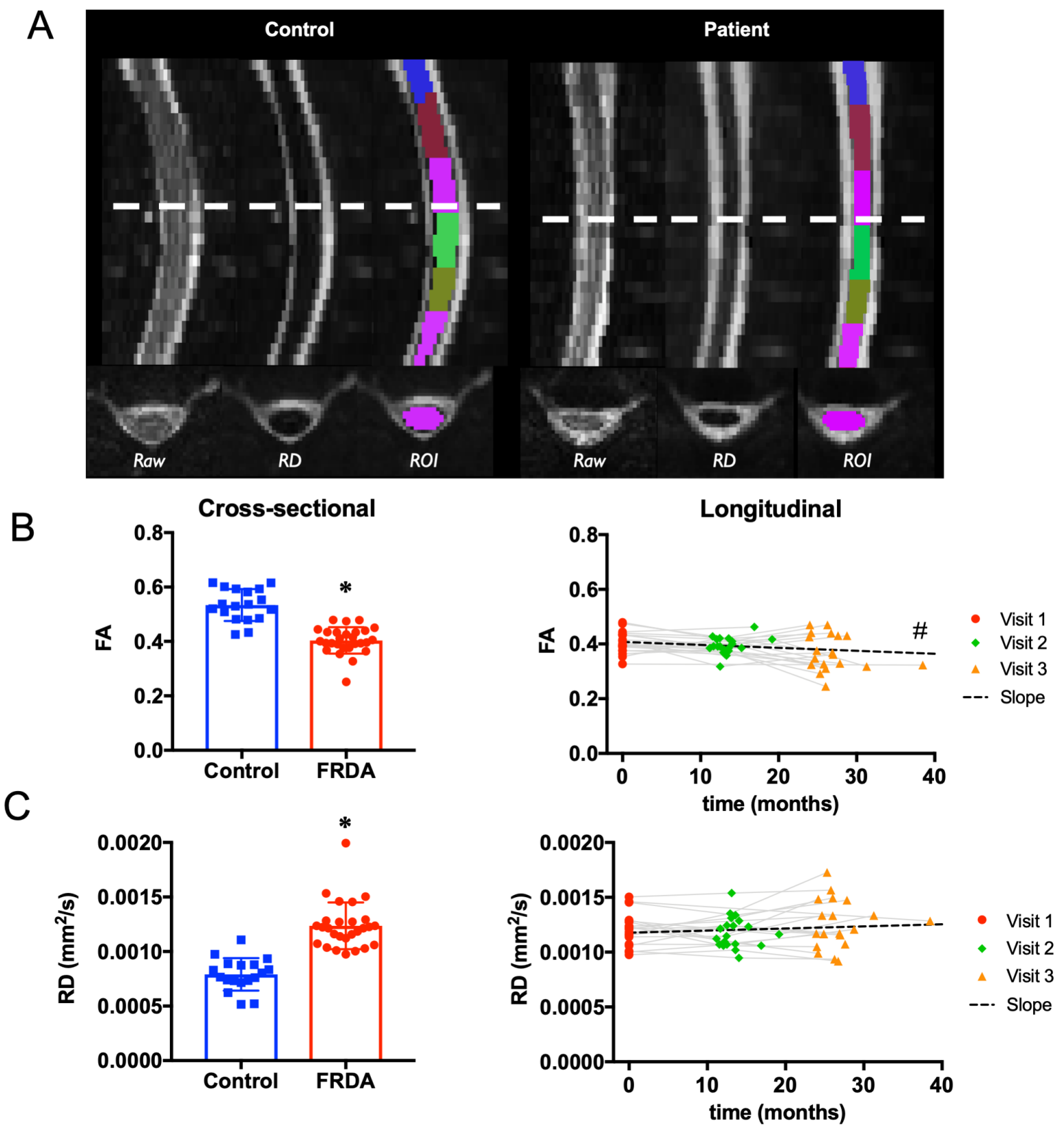

Figure 2. Spinal cord diffusion. (A) Diffusion images from a control participant (left), and FRDA participant (right) showing the raw image, the MD image and the ROI segmentation for each participant. (B) Left: Cross-sectional comparison of fractional anisotropy (FA) at C2-C3 in FRDA vs. Control at baseline. Right: Longitudinal change in FA at C2-C3 over time in FRDA. (C) Left: Cross-sectional comparison of mean diffusivity (MD) at C2-C3 in FRDA vs. Control at baseline. Right: Longitudinal change in MD at C2-C3 over time in FRDA. * FRDA statistically different from control. \# slope statistically $<0$. 
A

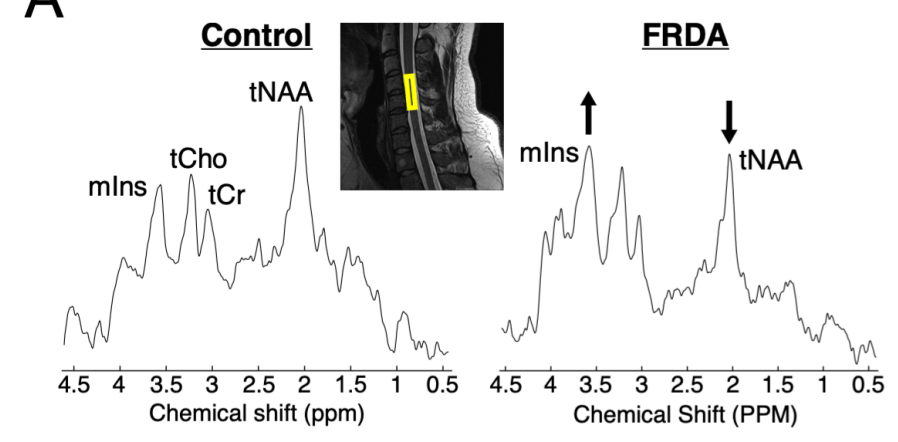

B

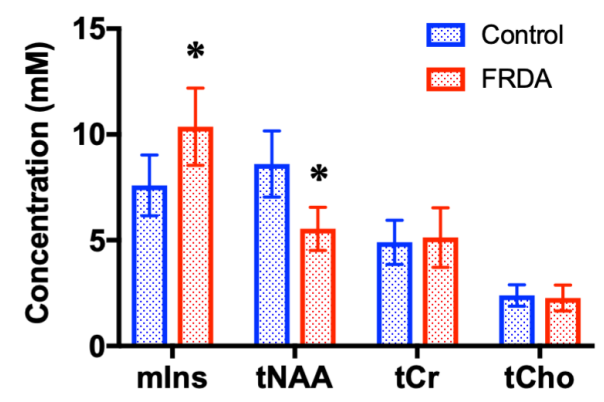

C

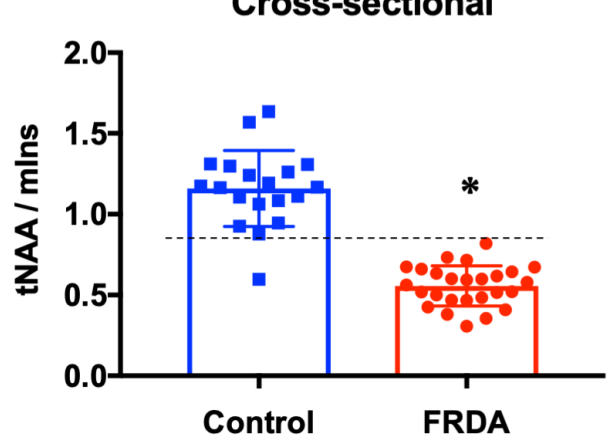

Longitudinal

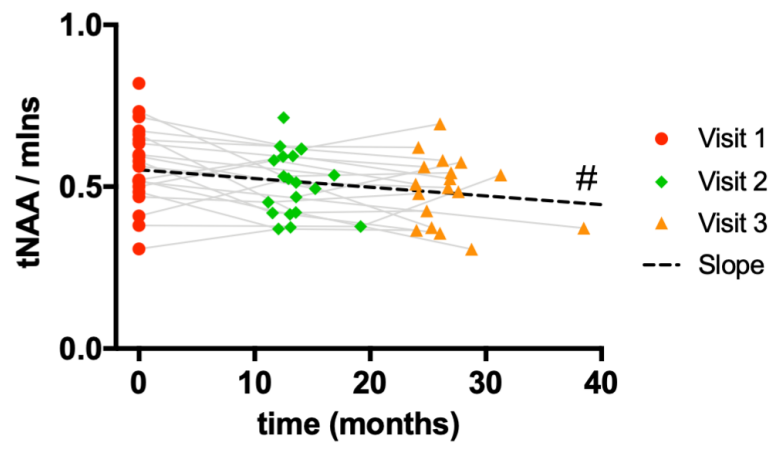

Figure 3. Spinal cord spectroscopy. (A) MR spectra from a control participant (left), and FRDA participant (right) clearly showing a lower $N$ NAA peak and a higher mIns peak in FRDA. The inset shows the 8x6x30mm voxel at C4-C5 on a T2 image. (B) Cross-sectional comparison of the concentration of the four main metabolites (mIns, $t N A A, t C r$ and tCho) in FRDA vs. Control at baseline. (C) Left: Crosssectional comparison of the ratio $t N A A / m I n s$ at C4-C5 in FRDA vs. Control at baseline. Right: Longitudinal change in $\mathrm{tNAA}$ mIns at C4-C5 over time in FRDA. * FRDA statistically different from control, \# slope statistically $<0$. 


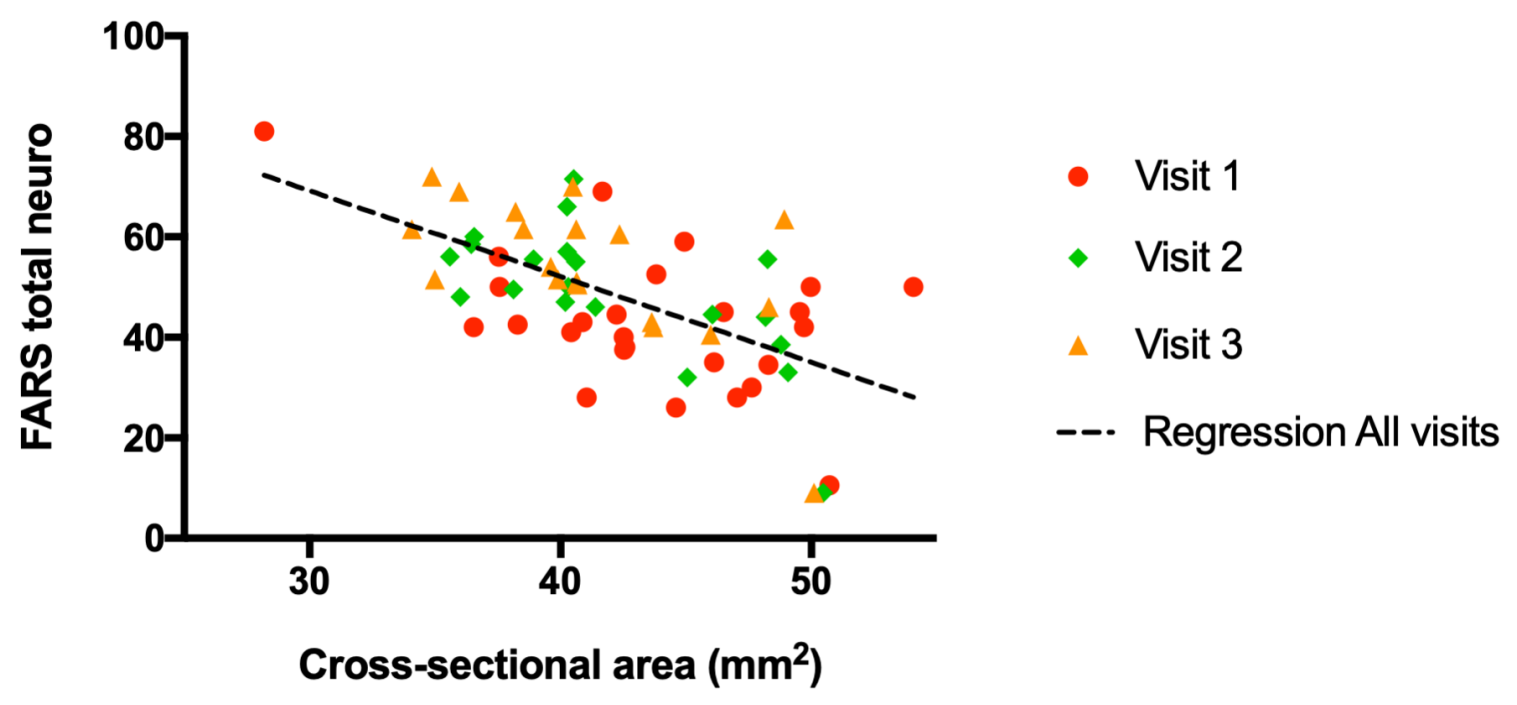

Figure 4. Correlation between FARS total neuro and cross-sectional area. Correlation was determined using cross-sectional area as predictor variable and FARS total neuro as response variable. Data from all three visits were used. $R=-0.60, p<7.10^{-6}$ 
medRxiv preprint doi: https://doi.org/10.1101/2022.01.28.22270048; this version posted January 30, 2022. The copyright holder for this preprint (which was not certified by peer review) is the author/funder, who has granted medRxiv a license to display the preprint in perpetuity.

It is made available under a CC-BY-NC-ND 4.0 International license .

\section{TABLES}

\begin{tabular}{|c|c|c|c|c|c|}
\hline & \multicolumn{2}{|c|}{ Cross-sectional cohort } & \multicolumn{3}{|c|}{ Longitudinal cohort (FRDA only) } \\
\hline & CTRL & FRDA & Baseline & Iy follow-up & $2 y$ follow-up \\
\hline Number of subjects & 20 (I IM/9F) & $28(\mathrm{I} 4 \mathrm{M} / \mathrm{I} 4 \mathrm{~F})$ & $2 I(I O M / I I F)$ & $2 I(I 0 M / I I F)$ & $19(9 \mathrm{M} / \mathrm{IOF})$ \\
\hline Age (years) & $20.4(7.1)$ & $19.0(7.3)$ & $19.0(7.7)$ & $20.1(7.7)$ & $21.5(8.0)$ \\
\hline Age of onset (years) & - & $13.4(5.2)$ & $13.8(5.8)$ & $13.8(5.8)$ & I4.I (6.0) \\
\hline Disease duration (years) & - & $5.5(4.0)$ & $5.1(3.9)$ & $6.3(3.9)$ & $7.4(4.2)$ \\
\hline Time from diagnosis (years) & - & $2.3(2.6)$ & $2.1(2.9)$ & $3.2(2.9)$ & $4.2(3.0)$ \\
\hline GAA repeats (shorter) ${ }^{a}$ & - & 597 (I79) & $598(191)$ & $598(191)$ & $596(200)$ \\
\hline GAA repeats (longer) & - & $959(205)$ & $987(221)$ & $987(221)$ & $960(197)$ \\
\hline SARA $(0-40)^{b}$ & $0(0)$ & $8.9(2.8)$ & $9.6(2.8)$ & II.6 (4.5) & $13.8(5.2)$ \\
\hline FARS score neuro $(0-1 \mid 7)$ & $0(0)$ & $42.7(13.6)$ & $42.6(11.8)$ & $48.7(13.3)$ & $53.9(14.5)$ \\
\hline Functional Staging (0-6) & $0(0)$ & $2.0(1.0)$ & $1.9(1.0)$ & $2.5(1.1)$ & $2.8(1.0)$ \\
\hline ADL (0-36) & $0(0)$ & $5.8(3.5)$ & $5.4(2.9)$ & $8.0(3.6)$ & $9.9(4.0)$ \\
\hline 9HPT dominant (s) & $19.2(2.8)$ & $40.4(12.0)$ & $38.8(9.8)$ & $41.0(11.2)$ & $41.5(10.7)$ \\
\hline 9HPT non-dominant (s) & $19.2(2.4)$ & $43.4(13.0)$ & $43.1(11.2)$ & $47.8(13.0)$ & $50.2(10.7)$ \\
\hline Timed walk (s)c & $4.3(0.6)$ & $9.4(19.1)$ & $5.8(0.9)$ & $5.8(1.4)$ & $6.0(1.5)$ \\
\hline Time from baseline visit (months) & 0 & 0 & 0 & $13.7(2.2)$ & $26.8(3.4)$ \\
\hline
\end{tabular}

Table I. Cohort demographic and clinical characteristics at baseline.

Aside from numbers of subjects, values shown are mean (standard deviation).

a One participant had a point mutation in one of the alleles. The shorter GAA value was left blank.

b 15 out of 28 FRDA participants in the cross-sectional cohort, including 10 out of $2 \mathrm{I}$ in the longitudinal cohort, have no SARA score at baseline because SARA was added to the protocol after they were recruited.

c One participant was unable to walk (no value), and another completed the walk in 105s (outlier excluded).

\begin{tabular}{|c|c|c|c|c|c|c|c|c|}
\hline & Modality & $\begin{array}{c}\text { CTRL } \\
\text { mean (sd) }\end{array}$ & $\begin{array}{c}\text { FRDA } \\
\text { mean (sd) }\end{array}$ & Cohen's d & $\begin{array}{l}\text { Difference } \\
\text { (\%) }\end{array}$ & Raw p & Corrected $\mathrm{p}$ & \\
\hline CSA $\left(\mathrm{mm}^{2}\right)(\mathrm{C} 2-\mathrm{C} 3)$ & Morphometry & $63.2(4.2)$ & $43.7(5.5)$ & -3.9 & $-31 \%$ & $2 \mathrm{E}-17$ & $4 \mathrm{E}-17$ & *** \\
\hline Eccentricity (C2-C3) & Morphometry & $0.75(0.05)$ & $0.83(0.04)$ & 1.7 & $10 \%$ & $5 \mathrm{E}-07$ & $5 \mathrm{E}-07$ & $* * *$ \\
\hline FA (C3-C6) & Diffusion & $0.53(0.06)$ & $0.40(0.05)$ & -2.5 & $-24 \%$ & $3 \mathrm{E}-10$ & IE-09 & $* * *$ \\
\hline$M D(C 3-C 6)\left(10^{-3} \mathrm{~mm}^{2} / \mathrm{s}\right)$ & Diffusion & $1.17(0.18)$ & $1.58(0.20)$ & 2.1 & $35 \%$ & 7E-09 & IE-08 & $* * *$ \\
\hline $\mathrm{RD}(\mathrm{C} 3-\mathrm{C} 6)\left(10^{-3} \mathrm{~mm}^{2} / \mathrm{s}\right)$ & Diffusion & $0.79(0.15)$ & $1.24(0.21)$ & 2.3 & $56 \%$ & $6 \mathrm{E}-10$ & $2 \mathrm{E}-09$ & $* * *$ \\
\hline$A D(C 3-C 6)\left(10^{-3} \mathrm{~mm}^{2} / \mathrm{s}\right)$ & Diffusion & $1.93(0.28)$ & $2.26(0.19)$ & 1.4 & $17 \%$ & $4 \mathrm{E}-05$ & $4 \mathrm{E}-05$ & $* * *$ \\
\hline tNAA/mIns (C4-C5) & Spectroscopy & $1.16(0.23)$ & $0.56(0.12)$ & -3.4 & $-52 \%$ & $4 \mathrm{E}-14$ & $2 \mathrm{E}-13$ & $* * *$ \\
\hline tNAA (C4-C5) (mM) & Spectroscopy & $8.6(1.6)$ & $5.5(1.0)$ & -2.4 & $-36 \%$ & IE-09 & $6 \mathrm{E}-09$ & $* * *$ \\
\hline mlns (C4-C5) (mM) & Spectroscopy & $7.6(1.4)$ & $10.4(1.8)$ & 1.7 & $37 \%$ & $8 \mathrm{E}-07$ & $2 \mathrm{E}-06$ & $* * *$ \\
\hline $\operatorname{tCr}(\mathrm{C} 4-\mathrm{C5})(\mathrm{mM})$ & Spectroscopy & $4.9(1.0)$ & $5.1(1.4)$ & 0.2 & $5 \%$ & 0.49 & 0.98 & ns \\
\hline tCho (C4-C5) (mM) & Spectroscopy & $2.4(0.5)$ & $2.3(0.6)$ & -0.2 & $-5 \%$ & 0.53 & 0.98 & ns \\
\hline
\end{tabular}

Table 2. Cross-sectional differences in the main morphometry, diffusion and spectroscopy parameters at baseline. $s d=$ standard deviation. $* * * \mathrm{p}<0.0005$ 
medRxiv preprint doi: https://doi.org/10.1101/2022.01.28.22270048; this version posted January 30, 2022. The copyright holder for this preprint (which was not certified by peer review) is the author/funder, who has granted medRxiv a license to display the preprint in perpetuity.

It is made available under a CC-BY-NC-ND 4.0 International license .

\begin{tabular}{|c|c|c|c|c|c|c|c|c|}
\hline & Modality & $\mathbf{N}$ & $\begin{array}{l}\text { Annual slope } \\
\text { mean (sd) }\end{array}$ & SRM & $\begin{array}{c}\text { Annual \% change } \\
\text { from baseline }\end{array}$ & Raw p & Corrected p & \\
\hline CSA (C2-C3) $\left(\mathrm{mm}^{2}\right)$ & Morphometry & 20 & $-1.03(1.09)$ & -0.95 & $-2.4 \%$ & $2 \mathrm{E}-04$ & $4 \mathrm{E}-04$ & $* * *$ \\
\hline Eccentricity (C2-C3) & Morphometry & 20 & $0.0013(0.0061)$ & 0.21 & $0.2 \%$ & 0.18 & 0.18 & ns \\
\hline tNAA/mIns & Spectroscopy & 20 & $-0.032(0.062)$ & -0.51 & $-5.8 \%$ & 0.02 & 0.03 & * \\
\hline FA (C3-C6) & Diffusion & 20 & $-0.013(0.026)$ & -0.50 & $-3.2 \%$ & 0.02 & 0.08 & trend \\
\hline$M D(C 3-C 6)\left(10^{-3} \mathrm{~mm}^{2} / \mathrm{s}\right)$ & Diffusion & 20 & $0.015(0.085)$ & 0.18 & $1.0 \%$ & 0.22 & 0.44 & ns \\
\hline $\mathrm{RD}(\mathrm{C} 3-\mathrm{C} 6)\left(10^{-3} \mathrm{~mm}^{2} / \mathrm{s}\right)$ & Diffusion & 20 & $0.023(0.091)$ & 0.25 & $1.9 \%$ & 0.14 & 0.42 & ns \\
\hline$A D(C 3-C 6)\left(10^{-3} \mathrm{~mm}^{2} / \mathrm{s}\right)$ & Diffusion & 20 & $-0.007(0.103)$ & -0.06 & $-0.3 \%$ & 0.39 & 0.44 & ns \\
\hline SARA & Clinical & 19 & $2.0(1.1)$ & 1.89 & & $8 \mathrm{E}-08$ & $4 \mathrm{E}-07$ & **** \\
\hline FARS total neuro & Clinical & 21 & $5.3(3.7)$ & 1.44 & & IE-06 & $3 \mathrm{E}-06$ & $* * *$ \\
\hline Functional staging & Clinical & 21 & $0.4(0.3)$ & 1.49 & & $6 \mathrm{E}-07$ & $3 E-06$ & $* * *$ \\
\hline ADL & Clinical & 21 & $2.0(1.4)$ & 1.39 & & $2 \mathrm{E}-06$ & $3 \mathrm{E}-06$ & $* * *$ \\
\hline 9HPT (non dom.) & Clinical & 21 & $3.4(4.0)$ & 0.86 & & $4 \mathrm{E}-04$ & $4 \mathrm{E}-04$ & $* * *$ \\
\hline
\end{tabular}

Table 3. Longitudinal annual change (slope) in the main MR and clinical parameters. $s d=$ standard deviation. SRM = Standardized Response Mean. * $p<0.05$, ** $p<0.01$, *** $p<0.001$

\begin{tabular}{lccccc}
\hline & SARA & FARS total neuro & Functional staging & ADL & 9HPT (non dom.) \\
\hline CSA (C2-C3) & $-0.55 * * *$ & $-0.60 * * *$ & $-0.48 * * *$ & $-0.51 * * *$ & $-0.42 * *$ \\
Eccentricity (C2-C3) & $-0.18 \mathrm{~ns}$ & $-0.25 \mathrm{~ns}$ & $-0.05 \mathrm{~ns}$ & $-0.19 \mathrm{~ns}$ & $-0.14 \mathrm{~ns}$ \\
\hline tNAA/mIns (C4-C5) & $-0.16 \mathrm{~ns}$ & $0.02 \mathrm{~ns}$ & $-0.17 *$ & $-0.31 * *$ & $-0.16 * *$ \\
\hline FA (C3-C6) & $-0.26 \mathrm{~ns}$ & $-0.30 * *$ & $-0.19 *$ & $-0.46 * *$ & $-0.22 \mathrm{~ns}$ \\
MD (C3-C6) & $0.17 \mathrm{~ns}$ & $0.22 \mathrm{~ns}$ & $0.12 \mathrm{~ns}$ & $0.19 \mathrm{~ns}$ & $0.16 \mathrm{~ns}$ \\
RD (C3-C6) & $0.04 \mathrm{~ns}$ & $0.09 \mathrm{~ns}$ & $0.00 \mathrm{~ns}$ & $-0.05 \mathrm{~ns}$ & $0.06 \mathrm{~ns}$ \\
AD (C3-C6) & $0.21 \mathrm{~ns}$ & $0.26 \mathrm{~ns}$ & $0.17 \mathrm{~ns}$ & $0.29 \mathrm{~ns}$ & $0.19 \mathrm{~ns}$ \\
\hline
\end{tabular}

Table 4. Regression coefficients between clinical parameters and MR parameters. Regressions were performed using data from all 3 time points. Numbers indicate the R coefficient between the corresponding clinical metric (response variable) and MR metric (predictor variable) Blue color indicates negative correlation and red color indicates positive correlation. Higher color intensity indicates higher absolute correlation coefficient $* \mathrm{p}<0.05, * * \mathrm{p}<0.005, * * * \mathrm{p}<0.0005$ 